\title{
Clinical experience with temporary right ventricular mechanical circulatory support
}

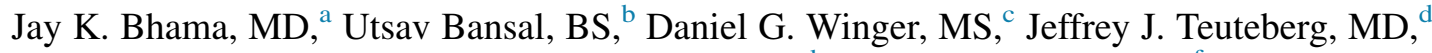

Christian Bermudez, MD, ${ }^{\mathrm{e}}$ Robert L. Kormos, $\mathrm{MD},{ }^{\mathrm{d}}$ and Aditya Bansal, $\mathrm{MD}^{\mathrm{f}}$

\section{ABSTRACT}

Objectives: This study sought to determine if indication for support affects the outcomes after temporary right ventricular mechanical circulatory support after postcardiotomy cardiogenic shock, cardiac transplant, or left ventricular assist device placement.

Methods: A retrospective review was performed on 80 patients receiving a right ventricular assist device. Data were collected from a prospectively maintained database. Kaplan-Meier survival analysis was performed to compare survival between groups. Multivariate regression analysis was performed to identify risk factors for failure to wean from support.

Results: The indication for support was postcardiotomy cardiogenic shock in 13 patients $(16 \%)$, cardiac transplant in 25 patients $(31 \%)$, and left ventricular assist device in 42 patients (53\%). Median support time was 6 days. Device was successfully weaned in 6 postcardiotomy cardiogenic shock cases $(46 \%), 21$ cardiac transplant cases $(84 \%)$, and 35 left ventricular assist device cases (83\%). Survival was worse for patients with postcardiotomy cardiogenic shock compared with patients with a left ventricular assist device. Survival up to 3 months was better for patients who received immediate $(\mathrm{n}=43)$ versus delayed $(\mathrm{n}=37)$ support $(79 \%$ vs $46 \%, P=.003)$. Weaning and survival remained static across implant era. Risk factor analysis identified postcardiotomy cardiogenic shock indication (odds ratio, $0.161 ; P=.007$; confidence interval, 0.043-0.600) as an independent negative predictor of weaning from mechanical support.

Conclusions: Temporary right ventricular mechanical support remains an effective treatment strategy after left ventricular assist device placement with immediate support resulting in superior short-term survival. Caution should be applied in postcardiotomy cardiogenic shock when weaning and survival are poor. Overall survival outcomes have remained relatively static over time. (J Thorac Cardiovasc Surg 2018;156:1885-91)

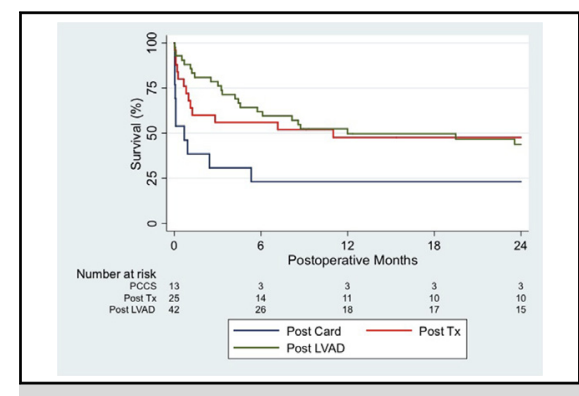

Kaplan-Meier survival analysis of study patients based on indication for RV support.

\section{Central Message}

Outcomes after temporary RV MCS vary according to indication (ie, PCCS, CTx, durable LVAD) and timing of support (ie, immediate vs delayed).

\section{Perspective}

RVF requiring mechanical support remains a formidable problem with evolving strategies for treatment. We found that outcomes are dependent on the indication for (ie, postcardiotomy vs CTx vs LVAD) and timing (immediate vs delayed) of support. Postcardiotomy indication was an independent predictor of weaning failure. Overall, outcomes have remained static over time, demonstrating the need for further research in this area.

See Editorial Commentary page 1892

See Editorial page 1881.

\footnotetext{
From the a Division of Cardiothoracic Surgery, University of Iowa Health Care, Iowa City, Iowa; ${ }^{\mathrm{b}}$ University of Pittsburgh School of Medicine, Pittsburgh, Pa; ${ }^{\mathrm{c}}$ Clinical and Translational Science Institute, University of Pittsburgh, Pittsburgh, Pa; ${ }^{\mathrm{d}} \mathrm{Heart}$ and Vascular Institute, Artificial Heart Program, University of Pittsburgh Medical Center, Pittsburgh, Pa; ${ }^{\mathrm{e}}$ Division of Cardiovascular Surgery, University of Pennsylvania, Philadelphia, $\mathrm{Pa}$; and ${ }^{\mathrm{f}}$ Division of Cardiothoracic Surgery, Ochsner Clinic, New Orleans, La.

Drs J. Bhama and U. Bansal contributed equally.

Received for publication July 9, 2017; revisions received April 17, 2018; accepted for publication April 20, 2018; available ahead of print June 1, 2018.

Address for reprints: Jay K. Bhama, MD, Division of Cardiothoracic Surgery, University of Iowa Health Care, 200 Hawkins Drive, Iowa City, IA 52242 (E-mail: jay-bhama@uiowa.edu).

$0022-5223 / \$ 36.00$

Copyright (c) 2018 by The American Association for Thoracic Surgery

https://doi.org/10.1016/j.jtcvs.2018.04.094
}

Right ventricular failure (RVF) continues to be a common clinical problem encountered in a variety of postoperative settings, including postcardiotomy cardiogenic shock (PCCS), cardiac transplant (CTx), and long-term therapy with a left ventricular assist device (LVAD). In such cases,

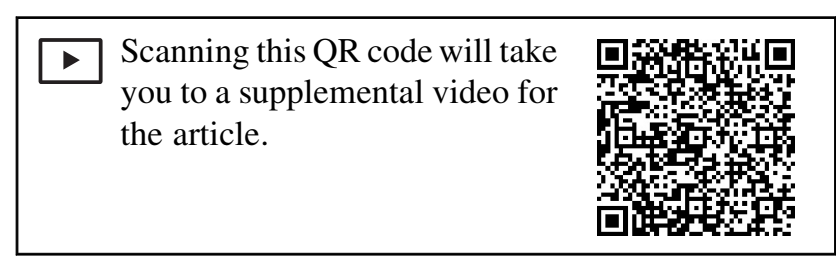




$$
\begin{aligned}
& \text { Abbreviations and Acronyms } \\
& \begin{aligned}
\mathrm{CI} & =\text { confidence interval } \\
\mathrm{CPB} & =\text { cardiopulmonary bypass } \\
\mathrm{CTx} & =\text { cardiac transplant } \\
\mathrm{LV} & =\text { left ventricular } \\
\mathrm{LVAD} & =\text { left ventricular assist device } \\
\mathrm{MCS} & =\text { mechanical circulatory support } \\
\mathrm{OR} & =\text { odds ratio } \\
\mathrm{PCCS} & =\text { postcardiotomy cardiogenic shock } \\
\mathrm{PVAD} & =\text { paracorporeal ventricular assist device } \\
\mathrm{RV} & =\text { right ventricle } \\
\mathrm{RVAD} & =\text { right ventricular assist device } \\
\mathrm{RVF} & =\text { right ventricular failure }
\end{aligned}
\end{aligned}
$$

use of temporary right ventricular (RV) mechanical circulatory support (MCS) may provide life-sustaining support allowing bridge to recovery or other intervention.

Our previous study examined the use of the CentriMag system (Thoratec Corp, Pleasanton, Calif) for temporary RV support in patients who developed RVF after the listed surgical procedures, ${ }^{1}$ providing a benchmark for comparing other modalities of RV support. ${ }^{2,3}$ The primary limitation of that initial study was a limited number of patients precluding comparison of outcomes across groups. The aim of the current study is to provide an update on our experience with temporary mechanical RV support in a variety of surgical settings with a larger cohort of patients (Video 1). This may be particularly relevant in light of the new heart transplant allocation policies that may prioritize patients supported with surgical temporary mechanical support devices.

\section{MATERIALS AND METHODS \\ Patients}

Permission was obtained from the University of Pittsburgh Medical Center Internal Review Board to access the prospectively collected database on patients who received RV MCS at the University of Pittsburgh Medical Center Presbyterian Hospital. We performed a retrospective review of 80 patients who underwent implantation of a right ventricular assist device (RVAD) due to RVF in the setting of PCCS, CTx, or LVAD between 2005 and 2013. One patient required device replacement within 12 hours of RVAD explantation. Patients who received ECMO were excluded. A complete review of the medical record was performed for each patient, including demographic, procedural, and outcome data. Adverse events after implantation were defined according to Interagency Registry for Mechanically Assisted Circulatory Support guidelines.

\section{Device Placement}

Transesophageal echocardiography was used to diagnose significant RV dysfunction despite inotropic support and inhaled nitric oxide. All patients received a CentriMag RVAD (St Jude Medical, St Paul, Minn, formerly Thoratec Corp), the majority through a midsternotomy with cardiopulmonary bypass (CPB) support using techniques previously described. ${ }^{1}$ One patient underwent placement of a percutaneous RVAD with the cannulas placed in the right atrium via the right femoral vein $(25 \mathrm{~F}$ multistage venous cannula; Medtronic Inc, Minneapolis, Minn) and the main pulmonary

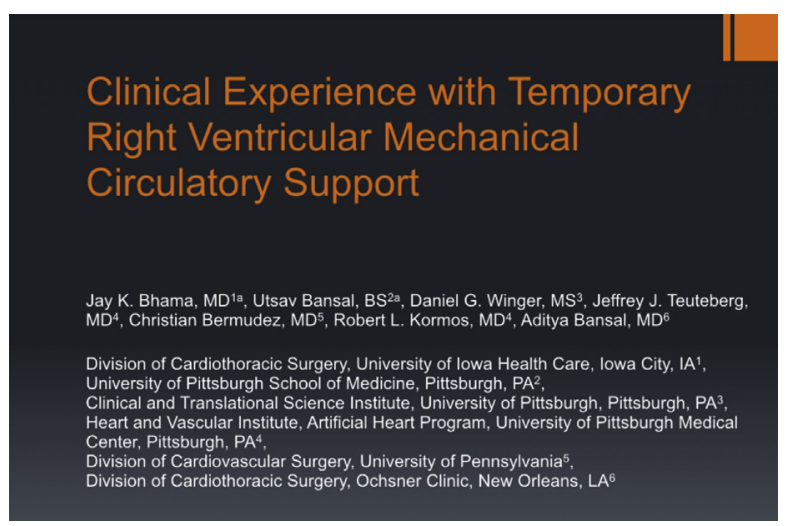

VIDEO 1. The importance and relevance of the present study. Video available at: https://www.jtcvs.org/article/S0022-5223(18)31233-9/fulltext.

artery via the right internal jugular vein $(17 \mathrm{~F}$ elongated venous cannula, Medtronic Inc) using fluoroscopic guidance.

The Centrimag RVAD was primed with normal saline, de-aired, and attached to the inflow and outflow cannulas. The pump speed was gradually increased to achieve flows of 4 to $6 \mathrm{~L} / \mathrm{min}$, with caution to avoid pulmonary overflow. The pulmonary artery diastolic pressures were monitored. As CentriMag flow was increased, CPB was weaned in a parallel fashion. Once CPB was removed, protamine sulfate was given to fully reverse the administered dose of heparin. Primary sternal closure was performed unless this was not possible because of excessive edema or bleeding, in which case the sternum was stented open with a rigid prosthesis and the skin was approximated or an Esmarch dressing was applied.

Patients receiving RVAD support during the same surgical setting as their primary procedure or within 24 hours of being indicated for RV support by hemodynamic or echocardiographic criteria were considered to have received immediate support. Those who received implants after 24 hours of being indicated for support were considered to have delayed support.

\section{Device Weaning and Removal}

Patients received a transthoracic echocardiogram in the intensive care unit 48 hours after RVAD implantation to evaluate RV functional status. Indications for improvement in RVF included increased amplitude of the pulmonary arterial waveform, decreased dependence on inotropic support, low central venous pressure, and improved RV systolic function on echocardiography. Before attempted weaning of the device, patients were administered low-dose inotropic support with milrinone $(\leq 0.25 \mu \mathrm{g} / \mathrm{kg} /$ $\min )$ or epinephrine $(\leq 0.05 \mu \mathrm{g} / \mathrm{kg} / \mathrm{min})$.

If RV function improved on the basis of the criteria listed, the patient was scheduled for elective Centrimag system removal in the operating room under the guidance of transesophageal echocardiogram. Patients in whom RV function did not improve 7 to 14 days postimplantation and were potential CTx candidates underwent exchange of device with a Thoratec paracorporeal ventricular assist device (PVAD) (Thoratec Corp) for long-term support. These patients were considered to have failed the weaning process. RV function was evaluated monthly.

\section{Anticoagulation}

Although blood products were given to normalize coagulation profile as needed, no anticoagulation was administered for the first 12 to 24 hours postoperatively. Once bleeding had decreased to less than $50 \mathrm{~mL} / \mathrm{h}$ from each chest tube for at least 4 to 6 hours, a heparin infusion was initiated to achieve an activated partial thromboplastin time of 59 to 72 seconds.

\section{Statistical Analysis}

Statistical analysis was performed using Stata 13 software (StataCorp LP, College Station, Tex) and SPSS Statistics 22.0 (IBM Corp, Armonk, 
NY). Descriptive statistics are reported as mean \pm standard deviation or mean with interquartile range. The analyses of survival time included pairwise comparison of the 3 groups, era analysis (surgery date 2005-2008 vs 2009-2013), early versus delayed implantation, and risk factor analysis for failure to wean. Actuarial survival estimates were calculated using KaplanMeier life-table analysis. A Bonferroni correction for $P$ values was applied when examining pairwise comparisons of 3 or more groups. Univariable and multivariable logistic regression analysis was performed to identify independent risk factors for successful weaning from RVAD support. Risk factors considered were age, sex, ischemic diagnosis, implant era, immediate institution of RVAD, PCCS indication, diabetes, postoperative bleeding requiring reoperation, arrhythmia, and stroke. A backward, stepwise analysis was used with a threshold for inclusion of $P<.10$. Results are demonstrated as odds ratios (OR), $95 \%$ confidence intervals (CIs), and $P$ value. A similar analysis was performed to identify risk factors for mortality. Risk factors considered in this analysis were age, sex, ischemic diagnosis, and indication for support.

\section{RESULTS}

\section{Demographics and Adverse Events}

Demographic data are summarized in Table 1. The indication for RV support was PCCS in 13 patients (16\%), CTx in 25 patients $(31 \%)$, and LVAD in 42 patients $(53 \%)$. The median support time was 6 days (interquartile range, 3-12). The overall rate of successful device weaning was 78\%: 6 PCCS cases (46\%), 21 CTx cases (84\%), and 35 LVAD cases $(83 \%)$ (Figure 1$)$. The most common postoperative adverse events included infection in 44 patients $(55 \%)$, reoperation for bleeding in 22 patients $(28 \%)$, and arrhythmia in 36 patients $(45 \%)$. These events are detailed according to indication for RV support in Table 2. There were no device failures.

\section{Device Weaning and Mortality}

Weaning failed in 18 patients (22\%): 7 PCCS (54\%), 4 CTx (16\%), and 7 LVAD (16\%). Although 6 of the patients with PCCS died within 3 days of RVAD implantation, 1 patient underwent device exchange for a Thoratec PVAD. The 4 patients who underwent CTx died while on the RVAD device, of whom 3 died within 8 days and the remaining patient died 38 days post-RVAD implantation. Of the 7 patients who failed to wean in the LVAD group, 3 received a Thoratec PVAD and 4 died on the device.

Early mortality (defined as $<30$ days or before discharge) for the entire cohort occurred in 29 patients $(36 \%)$, of whom 15 (19\%) died on RVAD support. Late death (defined as $>30$ days or after discharge) occurred in 17 patients $(21 \%)$. Overall survival was higher in the LVAD group than in the PCCS and CTx groups (Figure 1). The PCCS patient population had a sharp early drop-off with a survival of $38.5 \%$ after 1 month. According to Bonferroni adjusted pairwise comparisons between groups (Figure 2 inset), PCCS cases seemed to do significantly worse than LVAD cases $(P<.033)$. A significant survival difference could not be established for the CTx group compared with the LVAD or PCCS groups.

\section{Immediate Versus Delayed Right Ventricular Assist Device Support}

Kaplan-Meier survival according to timing of RVAD placement is shown in Figure 3. As demonstrated, overall survival at 3 months $(79 \%$ vs $46 \%, P=.003)$ was better

TABLE 1. Demographic characteristics of study patients based on indication for support

\begin{tabular}{|c|c|c|c|c|}
\hline Characteristic & $\operatorname{PCCS}(n=13)$ & CTx $(\mathbf{n}=25)$ & $\operatorname{LVAD}(n=42)$ & $P$ value \\
\hline Age, $y$ & $64 \pm 11$ & $59 \pm 10$ & $55 \pm 13$ & .06 \\
\hline Gender & & & & .03 \\
\hline Male $(\%)$ & $9(69)$ & $23(92)$ & $27(64)$ & \\
\hline Female $(\%)$ & $4(31)$ & $2(8)$ & $15(36)$ & \\
\hline Diagnosis $(\%)$ & & & & .01 \\
\hline Ischemic cardiomyopathy & $11(85)$ & $12(48)$ & $22(52)$ & \\
\hline Nonischemic cardiomyopathy & $0(0)$ & $10(40)$ & $18(43)$ & \\
\hline Other* & $2(15)$ & $3(12)$ & $2(5)$ & \\
\hline \multicolumn{5}{|l|}{ Comorbidities (\%) } \\
\hline Diabetes & $4(31)$ & $6(24)$ & $14(33)$ & .76 \\
\hline Hypertension & $9(69)$ & $13(52)$ & $21(50)$ & .47 \\
\hline \multicolumn{5}{|l|}{ Type of LVAD (\%) } \\
\hline HeartMate XVE (Thoratec Corp, Pleasanton, Calif) & - & - & $1(2)$ & \\
\hline Thoratec PVAD (Thoratec Corp) & - & - & $10(24)$ & \\
\hline Jarvik 2000 (Jarvik Heart Inc, New York, NY) & - & - & $1(2)$ & \\
\hline VentrAssist (Ventracor Ltd, Chatswood, Australia) & - & - & $5(12)$ & \\
\hline HeartMate II (Thoratec Corp) & - & - & $18(44)$ & \\
\hline HeartWare (Heartware Inc, Framingham, Mass) & - & - & $7(17)$ & \\
\hline Length of support (d) & $7 \pm 9$ & $10 \pm 11$ & $11 \pm 11$ & .56 \\
\hline
\end{tabular}

PCCS, Postcardiotomy cardiogenic shock; $C T x$, cardiac transplantation; $L V A D$, left ventricular assist device; $P V A D$, paracorporeal ventricular assist device. *These included valvular heart disease, congenital heart disease, massive pulmonary embolism, and postpartum cardiomyopathy. 


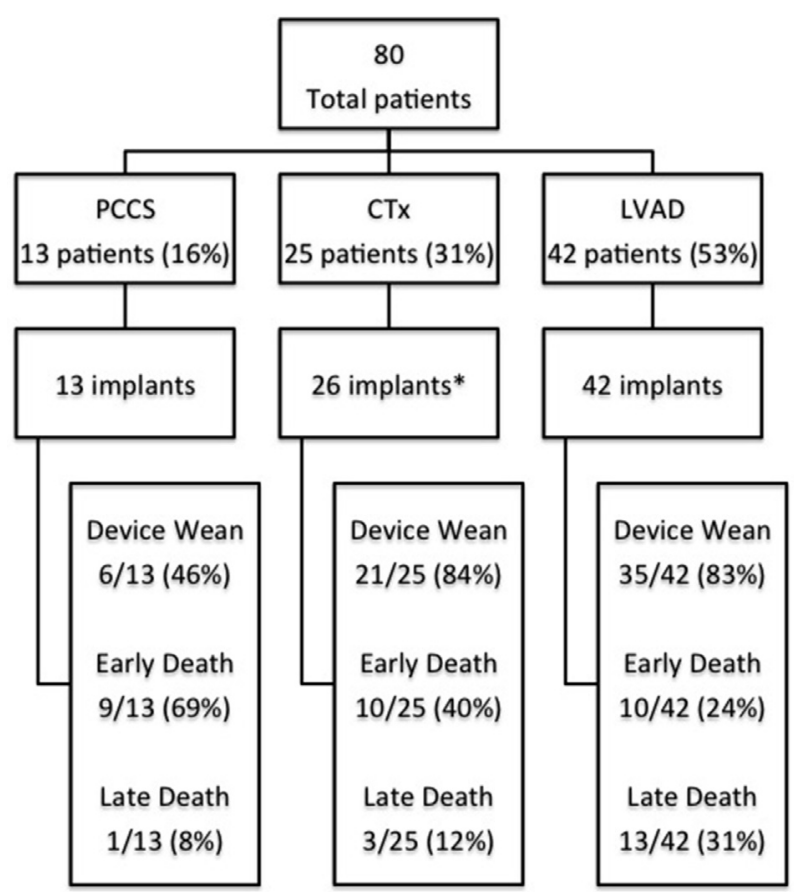

FIGURE 1. Flow diagram demonstrates outcomes according to the indication for RV support. *One patient underwent reinstitution of CentriMag system (Thoratec Corp, Pleasanton, Calif) RVAD support within 12 hours of device removal. PCCS, Postcardiotomy cardiogenic shock; $C T x$, cardiac transplantation; $L V A D$, left ventricular assist device.

for patients who received immediate $(\mathrm{n}=43)$ versus delayed $(\mathrm{n}=37)$ RVAD support, respectively. Among the 43 patients who received immediate support, $36(84 \%)$ were able to wean off of the device versus 27 of the 38 patients $(71 \%)$ who received delayed support $(P=.192)$.

\section{Era of Device Placement}

Although survival appears to be consistently higher in the 2009-2013 era versus the 2005-2008 era, there was no statistically significant improvement based on KaplanMeier survival analysis (Figure 4). Among the 42 patients who underwent RVAD placement in the recent era, 20 $(48 \%)$ received immediate support, whereas 23 of 39 patients $(59 \%)$ in the older generation received immediate support $(P=.375)$. In addition, there was also no significant improvement in weaning rates between the 2005-2008 era (28 patients, $72 \%$ ) and the $2009-2013$ era (34 patients, $83 \% P=.233)$.

\section{Risk Factors for Right Ventricular Assist Device Weaning Success and Mortality}

Univariable and multivariable logistic regression analyses for RVAD weaning success are shown in Table 3. Multivariable analysis identified PCCS indication (OR, $0.161 ; P=.007$; CI, 0.043-0.600) as an independent negative predictor of weaning from RVAD support. Female sex nearly approached significance $(\mathrm{OR}, 0.313 ; P=.056$; CI, 0.095-1.032). Regarding mortality, risk factor analysis failed to identify any significant predictors in this patient population.

\section{DISCUSSION}

This study provides further evidence supporting the use of temporary RV MCS as an effective treatment for postoperative RVF, especially in the CTx and LVAD population. Caution should be applied in the setting of PCCS where outcomes appear to remain relatively dismal. In updating our experience on the use of temporary MCS for RVF, we sought to answer 4 major questions: (1) Does indication for support affect survival or weaning rate, (2) does timing of support (immediate vs delayed implantation) affect survival or weaning rate, (3) has the survival or weaning rate improved over time, and (4) what are the factors contributing to weaning success/failure?

TABLE 2. Adverse events and causes of death in study patients

\begin{tabular}{|c|c|c|c|c|}
\hline Variable & $\operatorname{PCCS}(n=13),(\%)$ & CTx $(n=25),(\%)$ & $\operatorname{LVAD}(n=42),(\%)$ & $P$ value \\
\hline \multicolumn{5}{|l|}{ Adverse event } \\
\hline Reoperation for bleeding & $3(23)$ & $9(36)$ & $10(24)$ & .52 \\
\hline Major infection & $8(62)$ & $13(52)$ & $23(55)$ & .85 \\
\hline Arrhythmia & $5(38)$ & $10(40)$ & $21(50)$ & .64 \\
\hline Stroke/encephalopathy & $1(8)$ & $3(12)$ & $9(21)$ & .54 \\
\hline Air embolism & $0(0)$ & $0(0)$ & $1(2)$ & .63 \\
\hline \multicolumn{5}{|l|}{ Causes of early death } \\
\hline MSOF/sepsis & $1(8)$ & $5(20)$ & $3(7)$ & .25 \\
\hline LV failure & $1(8)$ & $1(4)$ & $0(0)$ & .22 \\
\hline Stroke & $0(0)$ & $0(0)$ & $0(0)$ & - \\
\hline Care withdrawn & $5(38)$ & $1(4)$ & $4(10)$ & .01 \\
\hline \multicolumn{5}{|l|}{ Causes of late death } \\
\hline Stroke & $0(0)$ & $0(0)$ & $0(0)$ & - \\
\hline Care withdrawn & $1(8)$ & $1(4)$ & $4(10)$ & .71 \\
\hline
\end{tabular}

PCCS, Postcardiotomy cardiogenic shock; $C T x$, cardiac transplantation; $L V A D$, left ventricular assist device; $M S O F$, multisystem organ failure; $L V$, left ventricular. 


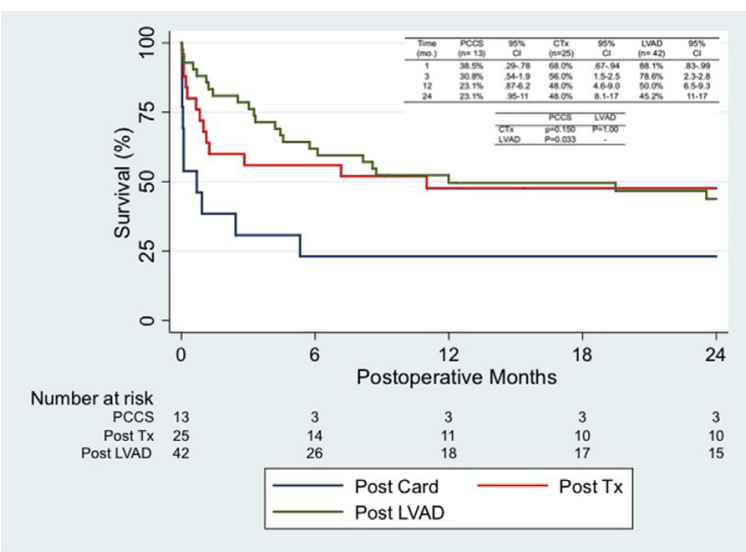

FIGURE 2. Kaplan-Meier survival analysis of study patients based on indication for RV support. PCCS, Postcardiotomy cardiogenic shock; $C I$, confidence interval; $C T x$, cardiac transplantation; $L V A D$, left ventricular assist device.

\section{Indication for Support}

In our previous publication, we concluded that the CentriMag system provides effective temporary MCS for acute RVF in a variety of settings with higher rates of RV recovery, weaning, and survival than previously reported. ${ }^{1}$ The study was insufficiently powered to identify a difference in survival or weaning rate based on the indication for support, although the data suggested a worse outcome in the PCCS cohort. The present study demonstrates a significant difference in survival outcome based on indication for support in patients with PCCS experiencing worse survival compared with patients supported after LVAD. Patients supported after CTx had intermediate survival between PCCS and LVAD cases, and statistical significance could not be achieved in comparison with either of the other 2 groups. We surmise that transplant recipients who develop RVF may be less likely to recover compared with LVAD recipients, because the cause of RV dysfunction is different in

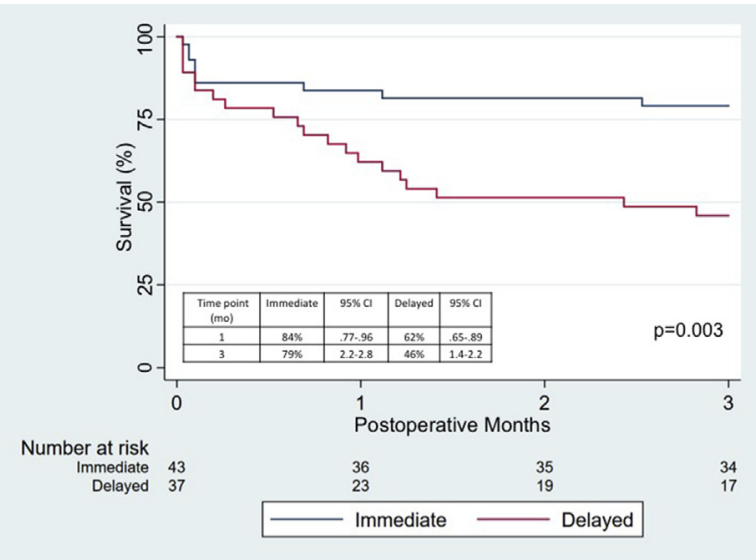

FIGURE 3. Kaplan-Meier survival analysis of study patients based on timing of implantation. $C I$, Confidence interval.

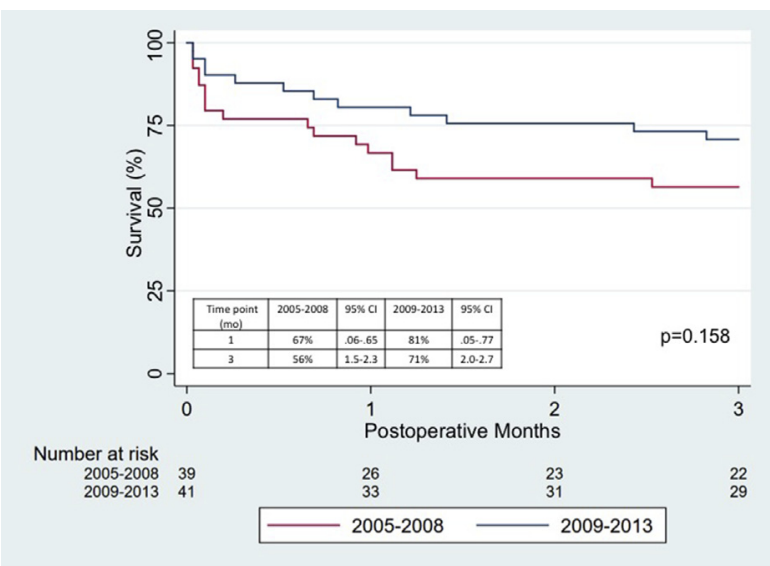

FIGURE 4. Kaplan-Meier survival analysis of study patients based on era of implantation. $C I$, Confidence interval.

these 2 settings. After CTx, RV dysfunction is often related to suboptimal myocardial protection, long ischemic times, and fixed degrees of pulmonary hypertension. RV dysfunction in this setting may not resolve as readily as when it is related to reversible pulmonary hypertension and septal shifting as seen in the post-LVAD setting, which are easier to modulate. ${ }^{4}$ An additional difference is that ongoing left ventricular (LV) dysfunction due to primary graft dysfunction in the post-transplant setting may contribute to ongoing RV dysfunction in the post-transplant setting, whereas LVAD replacement therapy usually abrogates any concern regarding $L V$ dysfunction.

We suspect that PCCS cases represent a higher risk cohort compared with CTx or LVAD cases. This is borne out by differences identified in comparing the basic demographics of the group of those with PCCS having older patients and a higher proportion of ischemic disease. Patients receiving CTx or LVAD are typically highly selected and younger, having less comorbidity compared with patients undergoing general cardiac surgery, who may be at extremes of age, frailty, and comorbid condition. PCCS cases also may have had significant variability in myocardial protection related to underlying coronary artery disease or need for concomitant valvular heart disease prolonging cardiac ischemic times. ${ }^{5}$ As opposed to patients receiving CTx and LVAD, who have correction of their LV dysfunction by LVAD or transplant, patients in the PCCS group continue to typically experience effects from their underlying level of LV dysfunction now potentially augmented by myocardial arrest with the usual sequela of compromised end-organ perfusion.

\section{Timing of Right Ventricular Support: Immediate Versus Delayed}

This study demonstrates increased short-term survival in patients who received immediate RVAD implantation 
TABLE 3. Univariable and multivariable logistic regression analysis of risk factors for successful weaning from right ventricular assist device support

\begin{tabular}{llll}
\hline \multicolumn{1}{c}{ Risk factor } & OR & $\boldsymbol{P}$ value & \multicolumn{1}{c}{$\mathbf{9 5} \% \mathbf{C I}$} \\
\hline Univariate analysis & & & \\
$\quad$ Age & 0.881 & .817 & $0.301-2.578$ \\
Implant era (recent) & 1.90 & .237 & $0.654-5.571$ \\
Female sex & 0.331 & .052 & $0.109-1.01$ \\
Immediate institution of & 2.17 & .156 & $0.744-6.366$ \\
$\quad$ RVAD & & & \\
Diabetes & 2.56 & .171 & $0.666-9.845$ \\
Ischemic diagnosis & 0.971 & .957 & $0.338-2.792$ \\
Reoperation for bleeding & 0.982 & .976 & $0.304-3.173$ \\
Postoperative stroke & 1.72 & .506 & $0.346-8.614$ \\
Postoperative arrhythmia & 1.87 & .262 & $0.625-5.629$ \\
$\quad$ PCCS indication & 0.168 & .006 & $0.047-0.598$ \\
Multivariate analysis & & & \\
PCCS indication & 0.161 & .007 & $0.043-0.60$ \\
Female sex & 0.313 & .056 & $0.095-1.03$ \\
\hline
\end{tabular}

$O R$, Odds ratio; $C I$, confidence interval; $R V A D$, right ventricular assist device; $P C C S$, postcardiotomy cardiogenic shock.

compared with delayed implantation. This is consistent with other studies that have shown that one of the most significant risk factors for mortality in patients receiving an LVAD is the development of RVF requiring an RVAD, ${ }^{6}$ and those patients with immediate biventricular assist device implantation have significant improvement in survival and ability to bridge to transplant. ${ }^{7,8}$

It is not surprising that short-term outcomes were improved with immediate support given the detrimental impact of RV dysfunction on end-organ perfusion in this patient population. RV dysfunction not only leads to poor $\mathrm{LV}$ filling and cardiac output but also contributes to elevated central venous pressures, tricuspid regurgitation, and endorgan congestion. The vicious cycles of this physiologic disarray compound the usual degree of preoperative endorgan dysfunction typically encountered in chronically ill patients with heart failure. ${ }^{9}$ In this analysis, we thought a focus on short-term outcome was a more appropriate reflection of the impact of temporary mechanical support as opposed to long-term outcomes because by 3 to 6 months most patients have died of RVF, recovered, or moved on to alternative therapeutic options, thereby mitigating a long-term impact on survival.

\section{Implantation Era}

We compared the use of RVADs for postoperative RVF in 2 eras of our clinical experience: 2005-2008 and 20092013. We hypothesized that short-term outcomes would have improved over time because of more timely recognition of RVF and improved management platforms in critical care. Although patients in the more recent era did have a slight increase in survival, the difference was insignificant and the overall outcomes in terms of survival and weaning rates have remained relatively static. In addition, the patient demographics and the use of immediate versus delayed implantation were similar between the 2 eras. Takeda and colleagues ${ }^{10}$ demonstrated an impact of era on survival in a cohort of LVAD cases requiring RV MCS with more recently implanted cases having improved survival. They identified that patients in the recent era were significantly less sick then patients in the previous era. They did not examine weaning success rates based on era. It is important to note that, in general, our group historically leaned toward earlier institution of RV support throughout both eras possibly mitigating a significant era effect. Patients in the recent era had a significant increase in number of support days (mean of 13.24 vs 6.82 days, $P=.004$ ), likely reflecting greater comfort with more protracted support times. Ultimately, this suggests that despite anticipated advancements in technology, surgical experience, and critical care management, other factors that likely contribute to the development of RV dysfunction, such as patient selection, donor selection, and LVAD device management, remain relatively static.

\section{Risk Factors for Weaning Success}

Given the markedly reduced weaning rate in the PCCS population compared with other indications for support, we were not surprised to find it to be a significant negative predictor of weaning success in multivariable analysis. We surmise that there are many explanations for this observation, as discussed earlier. However, in this broad population of postcardiac surgical patients receiving RV MCS, we were surprised to find that female sex was also a negative predictor of weaning success. There have been no previous studies, that we are aware of, evaluating risk factors for weaning success, and thus there is limited literature from which to draw comparisons. There have been studies that have identified gender differences in complication rates (ie, stroke, bleeding, pump thrombosis) in patients supported with LVADs; however, the impact of gender on overall outcomes in postsurgical patients remains poorly understood. ${ }^{11,12}$ Further investigation regarding how gender affects outcomes is warranted and should be the focus of future studies. It is interesting to note that immediate institution of RVAD support did not favor weaning success in this analysis. Although it was not found to be significant, there is certainly the clinical impression that early support is better. These data may be of particular relevance in the current era as heart transplant allocation policies shift toward prioritization of patient supported by temporary surgical mechanical support devices.

\section{Study Limitations}

This study has several limitations in addition to its retrospective nature. It is a single-center, retrospective, 
observational study, and despite having larger numbers than our previous study, it is still limited to some degree by sample size. Like other studies with small sample size, it is important to note that the absence of statistical significance does not infer the absence of clinical relevance as is true for our findings regarding weaning success and survival based on indication for support and era of implantation, which we believe to have clinical relevance. To further enhance the ability to detect differences among eras, indications, and immediate versus delayed implantation, multicenter studies or the use of a large registry database would be beneficial. The indication and timing for initiation of RV MCS were based on the individual patients and their managing physicians. There were no defined criteria for initiation of support. Therefore, it is likely that significant bias exists in patient selection. The risk factor analysis was also limited by a small number of patients, mandating us to limit the number of variables in the model and thus may not accurately reflect all factors contributing to weaning failure.

\section{CONCLUSIONS}

Temporary RV MCS continues to be an effective treatment for those patients in whom RVF develops, particularly in the setting of LVAD or CTx. Caution is warranted for patients with PCCS in whom outcomes remain consistently poor. Early detection of RVF and subsequent immediate RVAD implantation appears to provide benefit in the short term with respect to early survival and remains our recommended strategy. Female patients appear to be at particular risk for weaning failure, although little is known regarding why this is the case. Finally, despite obvious advancements in short-term mechanical support technology and management strategy, overall outcomes have remained relatively static over time, thus demonstrating the need for further investigation in the area of RVF.

\section{Conflict of Interest Statement}

Authors have nothing to disclose with regard to commercial support.

\section{References}

1. Bhama JK, Kormos RL, Toyoda Y, Teuteberg JJ, McCurry KR, Siegenthaler MP. Clinical experience using the Levitronix CentriMag system for temporary right ventricular mechanical circulatory support. J Heart Lung Transplant. 2009;28: 971-6.

2. Lampert BC, Teuteberg JJ. Right ventricular failure after left ventricular assist devices. J Heart Lung Transplant. 2015;34:1123-30.

3. Anderson MB, Goldstein J, Milano C, Morris LD, Kormos RL, Bhama J, et al. Benefits of a novel percutaneous ventricular assist device for right heart failure: the prospective RECOVER RIGHT study of the Impella RP device. J Heart Lung Transplant. 2015;34:1549-60.

4. Richmond ME, Easterwood R, Singh RK, Gilmore L, Beddows K, Zuckerman WA, et al. Low-dose donor dopamine is associated with a decreased risk of right heart failure in pediatric heart transplant recipients. Transplantation. 2016;100:2729-34

5. Kapur NK, Paruchuri V, Jagannathan A, Steinberg D, Chakrabarti AK, Pinto D, et al. Mechanical circulatory support for right ventricular failure. JACC Heart Fail. 2013;1:127-34.

6. Deng MC, Edwards LB, Hertz MI, Rowe AW, Keck BM, Kormos RL, et al. Mechanical circulatory support device database of the International Society for Heart and Lung Transplantation: Third annual report-2005. J Heart Lung Transplant. 2005;24:1182-7.

7. Fitzpatrick JR III, Frederick JR, Hiesinger W, Hsu VM, McCormick RC, Kozin ED, et al. Early planned institution of biventricular mechanical circulatory support results in improved outcomes compared with delayed conversion of a left ventricular assist device to a biventricular assist device. J Thorac Cardiovasc Surg. 2009;137:971-7.

8. Morgan JA, John R, Lee BJ, Oz MC, Naka Y. Is severe right ventricular failure in left ventricular assist device recipients a risk factor for unsuccessful bridging to transplant and post-transplant mortality. Ann Thorac Surg. 2004; 77:859-63.

9. Harjola VP, Mebazaa A, Čelutkiené J, Bettex D, Bueno H, Chioncel O, et al Contemporary management of acute right ventricular failure: a statement from the Heart Failure Association and the Working Group on Pulmonary Circulation and Right Ventricular Function of the European Society of Cardiology. Eur J Heart Fail. 2016;18:226-41.

10. Takeda K, Naka Y, Yang JA, Uriel N, Colombo PC, Jorde UP, et al. Timing of temporary right ventricular assist device insertion for severe right heart failure after left ventricular assist device implantation. ASAIO J. 2013;59: 564-9.

11. Morris AA, Pekarek A, Wittersheim K, Cole RT, Gupta D, Nguyen D et al. Gender differences in the risk of stroke during support with continuous-flow left ventricular assist device. J Heart Lung Transplant. 2015;34:1570-7.

12. Boyle AJ, Jorde UP, Sun B, Park SJ, Milano CA, Frazier OH, et al. Pre-operative risk factors of bleeding and stroke during left ventricular assist device support: an analysis of more than 900 HeartMate II outpatients. J Am Coll Cardiol. 2014;63: 880-8.

Key Words: LVAD, MCS, CentriMag, RVF, heart transplant, cardiogenic shock 